\title{
Modified PTS with Interleaving for PAPR Reduction of OFDM Signal with QPSK Subblocks
}

\author{
Pandurangan Mukunthan and Perumal Dananjayan
}

\begin{abstract}
Orthogonal Frequency-Division Multiplexing (OFDM) is a striking technique for achieving high-bit-rate wireless data transmission as it has the ability to cope with severe channel conditions. However, the potentially large Peak-To-Average Power Ratio (PAPR) has limited its application. Partial Transmit Sequence (PTS) is an attractive scheme for PAPR reduction without distortion, but to obtain preferable PAPR performance it needs many Inverse Fast Fourier Transforms (IFFTs) which results in high complexity. In this paper, a modified PTS scheme combined with interleaving for PAPR reduction using Quadrature Phase Shift Keying Modulation (QPSK) has been presented. The scheme is very efficient and avoids the use of any extra IFFTs as was done in PAPR reduction by ordinary PTS technique. The simulation result shows that PAPR performance is improved with the increase in number of subblocks.
\end{abstract}

Index Terms-OFDM, PAPR, interleaving, subblock partition scheme.

\section{INTRODUCTION}

The increased claims for wireless multimedia and interactive internet a service has enabled exhaustive research efforts on high-speed data transmission. The highest bit rates in commercially deployed wireless systems [1] are achieved by means of Orthogonal Frequency Division Multiplexing (OFDM) in wireless local-area networks (LANs) like Wi-Fi. OFDM has been adopted as the modulation method of choice for practically all the new wireless technologies being used and developed today. It is perhaps the most spectrally efficient method [2] discovered so far, and it mitigates the severe problem of multipath propagation that causes massive data errors and loss of signal in the microwave and UHF spectrum. The IEEE $802.11 \mathrm{a} / \mathrm{g} / \mathrm{n}$ standards and the wideband wireless metro-area network (MAN) technology WiMAX uses OFDM. 4G cellular technology standard Long-Term Evolution (LTE) [3] and power-line networking technology use OFDM. One of the first triumphant and most prevalent uses of OFDM was in data modems connected to telephone lines. A major confront for high-speed broadband mobile application is inter-symbol interference (ISI) due to

Manuscript received February 12, 2013; revises April 21, 2013. This work was supported in part by the U.S. Department of Commerce under Grant BS123456

P. Mukunthan is with the Computer Science and Engineering Department, Adhiparasakthi Engineering College, Melmaruvathur, India (e-mail: mukunthanece@pec.edu).

P. Dananjayan is with the Electronics and Communication Engineering Department, Pondicherry Engineering College, Pondicherry, India (e-mail pdananjayan@pec.edu). time-dispersive nature of the terrestrial radio channel.

In OFDM, the input data is divided among large number of closely spaced orthogonal carriers (frequency division multiplexing). Here only small amount of data is carried by each carrier, reducing the ISI significantly. Since OFDM is a multicarrier transmission technique, signals at different frequencies can be added up constructively, and this leads to high Peak to Average Power Ratio (PAPR). The maximal value of the PAPR grows with logarithm of number of subcarriers. The OFDM signal passed through a nonlinear device, such as a transmit power amplifier, may suffer significant spectral spreading and in-band distortion. Signals with a high PAPR require the power amplifier to operate with a large backoff from their peak power. This effectively limits the average transmission power due to the non-linearity of the power amplifier.

Techniques such as clipping, interleaving method, selective mapping (SLM), and partial transmit sequence (PTS) were proposed by researchers to control the PAPR of the transmitted signals in OFDM systems. Clipping [4] could be an effective technique for PAPR reduction, but it is a nonlinear process and may cause significant in-band distortion, which degrades the BER performance and out-of-band noise, which reduces the spectral efficiency. The interleaved partitioned ordinary PTS scheme [5] has the lowest computational complexity but it has the worst PAPR performance because the generated candidates are not fully independent. In SLM [6], one OFDM signal of the lowest PAPR is selected in a set of several signals containing the same information data. In PTS [7], the lowest PAPR signal is made by optimally phase combining the signal subblocks. SLM and PTS are very flexible schemes and have an effective performance of the PAPR reduction without any degradation. However, both these techniques require much system complexity and computational burden because of the many IFFT stages and complex optimization procedure. Other possible alternative solution is then to exploit other parameters of the OFDM signal. Modified PTS scheme [8] is proposed to lower the computational complexity while maintaining the similar PAPR reduction performance compared with the ordinary PTS scheme. To alleviate the problem of high complexity further an approach [9] has been proposed, in which real and imaginary parts are separately multiplied with phase factors, moreover PAPR is conjointly optimized in real and imaginary parts. In this paper, modified PTS combining the interleaved partitioning for reducing the PAPR of OFDM signals with QPSK subblocks is proposed.

The rest of this paper is organized as follows. Section II, briefly introduces PAPR of OFDM signals. The detailed description of the interleaving technique is shown in 
Section III. The simulation results and discussions are presented in Section IV. Finally, conclusions are drawn in Section V.

\section{PAPR OF OFDM SIGNALS}

In OFDM modulation technique, a block of $N$ data symbols, $X_{k}=\left(X_{0}, X_{1}, \ldots . X_{N-1}\right)$, is formed with each symbol modulating the corresponding subcarrier from a set of subcarriers. The transmitter block diagram is illustrated in Fig. 1 where the incoming data is first modulated by using QPSK modulation. An OFDM carrier signal is the sum of a number of orthogonal subcarriers, with baseband data on each subcarrier being independently modulated commonly using some type of quadrature amplitude modulation (QAM) or phase shift keying (PSK). While it is promising to transmit more bits per symbol, if the energy of the constellation is to remain the same, the points on the constellation must be closer together and the transmission becomes more susceptible to noise. This results in a higher bit error rate than for the lower order QAM variants. In this mode there is a balance between achieving higher data rates and maintaining a satisfactory bit error rate for any radio communications system. The $\mathrm{N}$ subcarriers are chosen to be orthogonal.

The complex baseband OFDM signal for $N$ subcarriers can be written as

$$
x(t)=\frac{1}{\sqrt{N}} \sum_{k=0}^{N-1} X_{k} e^{j 2 \pi k f_{0} t} \quad 0 \leq t \leq T
$$

Replacing $\mathrm{t}=\mathrm{n} T_{b}$, where $T_{b}=\mathrm{T} / \mathrm{N}$, gives the discrete time version denoted by

$$
x(n)=\frac{1}{\sqrt{N}} \sum_{k=0}^{N-1} X_{k} e^{j 2 \pi \mathrm{kn} / \mathrm{LN}} \quad n=0,1 \ldots, N L-1
$$

where $L$ is the oversampling factor.

The PAPR of the transmitted OFDM signal, $x(t)$, is the ratio of the maximum to the average power, and is given as

$$
P A P R=\frac{\max _{0 \leq t \leq T}|x(t)|^{2}}{E\left[\left|x(t)^{2}\right|\right]}
$$

where $E$ is the expectation operator.

The PAPR of the continuous-time OFDM signal cannot be precisely computed in the Nyquist sampling rate, which corresponds to $\mathrm{N}$ samples per OFDM symbol. In this case, signal peaks may be skipped and PAPR estimates are not precise. So, oversampling is necessary. From the central limit theorem [10], it follows that for large values of $\mathrm{N}$, the real and imaginary values of $x(t)$ becomes Gaussian distributed. The amplitude of the OFDM signal therefore has the Rayleigh distribution with zero mean and a variance of $N$ times the variance of one complex sinusoid. The PAPR of the OFDM signal is typically characterized by its complementary cumulative distribution function (CCDF).

The CCDF is the probability that the PAPR exceeds a certain threshold $\mathrm{PAPR}_{0}$, which can be expressed as [11]

$$
\operatorname{CCDF}(\operatorname{PAPR}(x(n)))=P_{r}(\operatorname{PAPR}(x(n)))>\operatorname{PAPR}_{0}
$$

Due to the independence of the N samples, the CCDF of the PAPR of a data block with Nyquist rate sampling is given by

$$
P=P_{r}\left(\operatorname{PAPR}(x(n))>\operatorname{PAPR}_{0}\right)=1-\left(1-e^{-P A P R_{0}}\right)^{N}
$$

This expression assumes that the $N$ time domain signal samples are mutually independent and uncorrelated and it is not accurate for a small number of subcarriers. The independent assumption in (5) is not true for the oversampling case. An empirical expression [12] for the CCDF of PAPR can be obtained by approximating the distribution for $\mathrm{N}$ subcarriers and oversampling by the distribution for $\mathrm{LN},\left(L_{1}\right)$ uncorrelated subcarriers without oversampling. Therefore, the CCDF of PAPR computed of the L-time oversampled OFDM signal can be rewritten as

$$
P=P_{r}\left(\operatorname{PAPR}(x(n))>\operatorname{PAPR}_{0}\right)=1-\left(1-e^{-\mathrm{PAPR}_{0}}\right)^{\mathrm{LN}}
$$

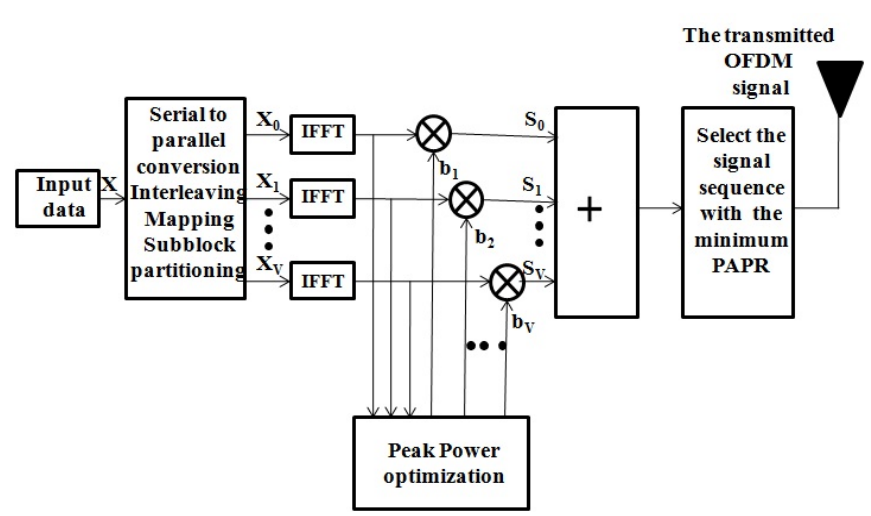

Fig. 1. A typical structure of transmitter for modified PTS combined with interleaved OFDM.

\section{INTERLEAVED OFDM}

Highly correlated data frames of OFDM signals have large PAPRs, which could be reduced, if the long correlation patterns are broken down. A set of fixed permutations (interleaving) is used in [13] this technique to break these correlation patterns. In this approach K-1 interleavers are used at the transmitter and these interleavers produce $\mathrm{K}-1$ permuted frames of the input data. The minimum PAPR frame of all the $\mathrm{K}$ frames is chosen for transmission. The uniqueness of the corresponding interleaver is also sent to the receiver as side information. Hence interleaving method [14] is simple to implement and reduces the transmitter complexity when compared with PTS scheme. If all the $K$, PAPR computations are done simultaneously and lowest PAPR sequence is selected in one step, the processing delay at the transmitter is significantly reduced. Therefore, it can also be used with high speed data transmissions.

Interleaved OFDM is also feasible for spectrum monitoring. Since subcarriers of one subblock are equally spaced [15], their frequency locations can be determined by capturing one subcarrier with the knowledge of system parameters. Users can monitor the radio activity on one subblock by sensing only one or two subcarriers of the 
subblock instead of all the subcarriers across the whole frequency band. Interleaving can be used to combat the effect of noise bursts and fading in error correction systems. By interleaving a data frame, the peaks in the associated OFDM signal can be compressed.

For Interleaved OFDM [16], the $\mathrm{N}$ subcarriers are partitioned into $V$ groups with each group having $Q$ contiguous subcarriers. Then the $k^{\text {th }}$ subcarrier of each group is assigned to the $k^{\text {th }}$ user.

$$
x^{(k)}(n)=\sum_{m=0}^{\mathrm{V}-1} X_{m}^{(k)} e^{j(2 \pi / N)(m Q+k) n}
$$

in which $k=0,1, \ldots . \mathrm{Q}-1$ is the index of users

Let $\left\{x_{n}: n=0,1, \ldots, N-1\right\}$ be data symbols to be modulated. Then, $\left\{X_{k}: k=0,1, \ldots, N-1\right\}$ are frequency domain samples after FFT of $\left\{x_{n}\right\},\left\{\tilde{X}_{l}: 1=0,1, \ldots, V-1\right\}$ are frequency domain samples after subcarrier mapping, and $\left\{\tilde{x}_{m}\right.$ : $m=0,1, \ldots, V-1\}$ are time domain symbols after IFFT of $\left\{\tilde{X}_{l}\right\}$ which can be described as follows

Let $\mathrm{m}=\mathrm{N}(q+n)$, where $0 \leq q \leq \mathrm{Q}-1$ and $0 \leq n \leq N-1$

Then,

$$
\begin{aligned}
\tilde{x}_{m}\left(=\tilde{x}_{N q+n}\right) & =\frac{1}{\mathrm{~V}} \sum_{l=0}^{\mathrm{V}-1} \tilde{X}_{l} e^{j 2 \pi \frac{m}{\mathrm{~V}} l}=\frac{1}{\mathrm{Q}} \cdot \frac{1}{N} \sum_{k=0}^{N-1} X_{k} e^{j 2 \pi \frac{m}{\mathrm{~V}} k} \\
& =\frac{1}{\mathrm{Q}} \cdot \frac{1}{N} \sum_{k=0}^{N-1} X_{k} e^{j 2 \pi \frac{N q+n}{N} k} \\
& =\frac{1}{\mathrm{Q}}\left(\frac{1}{\mathrm{~N}} \sum_{\mathrm{k}=0}^{\mathrm{N}-1} \mathrm{X}_{\mathrm{k}} \mathrm{e}^{\mathrm{j} 2 \pi \frac{\mathrm{n}}{\mathrm{N}} \mathrm{k}}\right) \\
& =\frac{1}{\mathrm{Q}} x_{n}
\end{aligned}
$$

where $l$ denotes a normalized discrete time instance, $q$ is the sub-channel index of the $k^{\text {th }}$ user and $\mathrm{N}$ is the total number of subcarriers

Here an $N$-sample interleaved OFDM block [17] is generated by repeating $l$ for $Q$ times. The resulting time symbols $\left\{\tilde{x}_{m}\right\}$ are simply a repetition of the original input symbols $\left\{x_{n}\right\}$ in the time domain. Therefore, an interleaved OFDM system with $N$ subcarriers can be scaled from an OFDM system.

\section{RESUlTS AND DisCUSSION}

The analysis of the modified PTS with interleaving method has been carried out using MATLAB 7.0. The simulation parameters considered for this analysis is summarized in Table I.

In the OFDM system under consideration, modified PTS technique is applied to the subblocks of uncoded information, which is modulated by QPSK modulation, and the phase rotation factors are transmitted directly to receiver through subblock. The performance evaluation is done in terms of complementary cumulative distribution function.

Fig. 2 depicts the variation of CCDF with PAPR of modified PTS with interleaving for different subblocks $V=2$,
4, 8, 16 when subcarriers $N=256$. From this figure it is shown that PAPR reduction performance increases with increase of subblocks.

TABLE I: SIMULATION PARAMETERS

\begin{tabular}{|l|l|c|}
\hline & $\begin{array}{l}\text { Simulation } \\
\text { Parameters }\end{array}$ & Type/Values \\
\hline \multirow{2}{*}{$\begin{array}{c}\text { Number of random } \\
\text { OFDM blocks }\end{array}$} & 10000 \\
\hline $\begin{array}{c}\text { Number of } \\
\text { subcarriers(N) }\end{array}$ & $256,512,1024$ \\
\hline & $\begin{array}{c}\text { Number of } \\
\text { subblocks(V) }\end{array}$ & $2,4,8,16$ \\
\hline & $\begin{array}{c}\text { Oversampling } \\
\text { factor(L) }\end{array}$ & Interleaving \\
\hline & $\begin{array}{c}\text { Subblock partition } \\
\text { scheme }\end{array}$ & QPSK \\
\hline & $\begin{array}{c}\text { Phase weighting } \\
\text { factor (b) }\end{array}$ & $1,-1, \mathrm{j},-\mathrm{j}$ \\
\hline
\end{tabular}

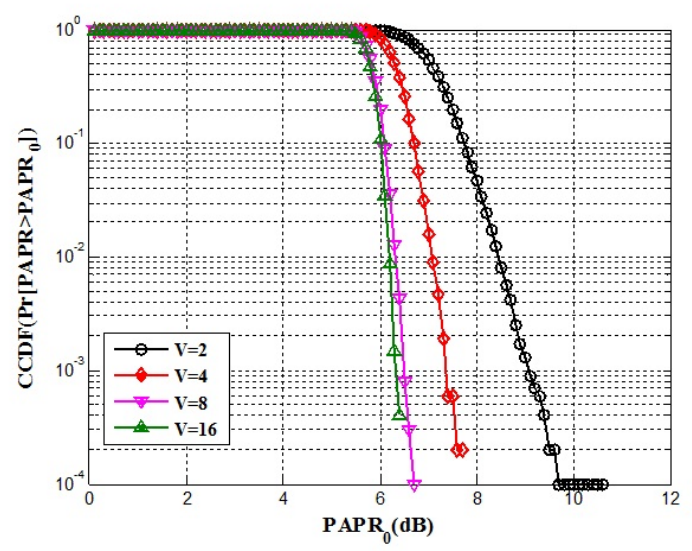

Fig. 2. CCDF of PAPR for different subblocks when $\mathrm{N}=256$ subcarriers.

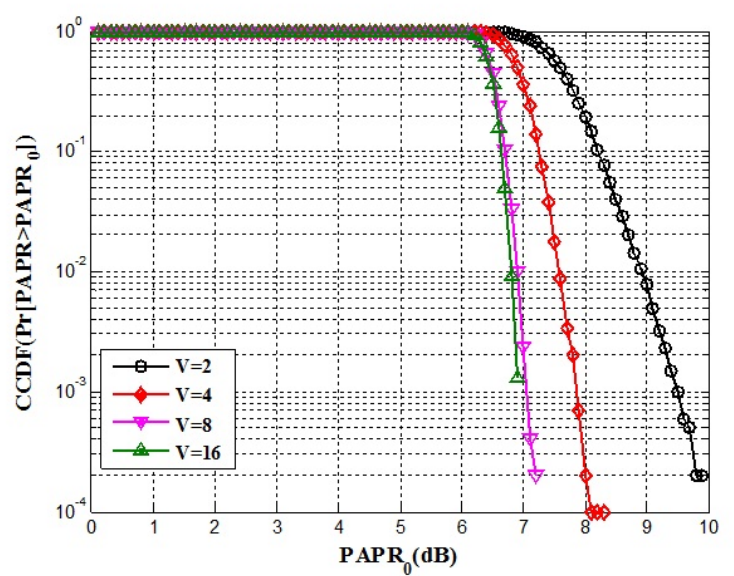

Fig. 3. CCDF of PAPR for different subblocks when $\mathrm{N}=512$ subcarriers.

The variation of CCDF with PAPR of modified PTS with interleaving for different subblocks $V=2,4,8,16$ when subcarriers $N=512$ is shown in Fig.3. By comparing the Figs. 2 and 3 it is evident that PAPR is increased from $7 \mathrm{~dB}$ to $7.6 \mathrm{~dB}$ when $V=4$ subblocks with number of subcarriers increased from $N=256$ to 512 at CCDF of $10^{-2}$.

Fig. 4 shows the variation of $\mathrm{CCDF}$ with PAPR of modified PTS with interleaving for different subblocks $V=2$, 
4, 8, 16 when subcarriers $N=1024$. From these Figs. 2, 3 and 4 it is concluded that PAPR decreases with increase of subblocks and increases with increase of subcarriers.

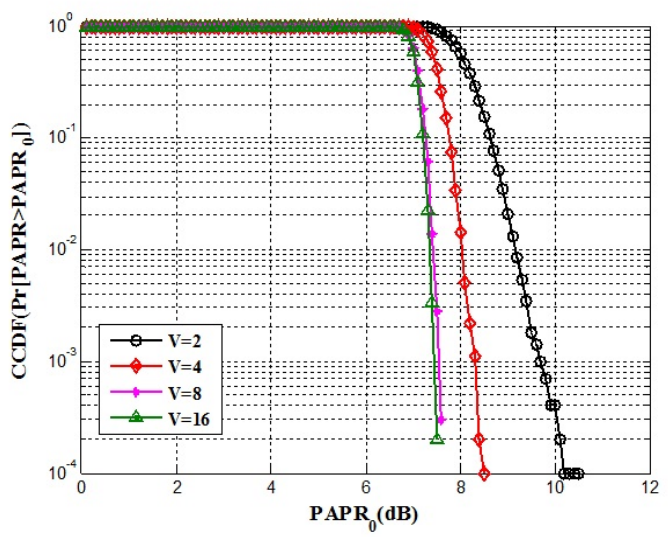

Fig. 4. $\mathrm{CCDF}$ of $\mathrm{PAPR}$ for different subblocks when $\mathrm{N}=1024$ subcarriers.

\section{CONCLUSION}

This paper discusses a modified PTS with interleaving and QPSK modulation to reduce the peak-to-average power ratio for OFDM transmission. The method used here avoids the need of any extra IFFTs as was done in PAPR reduction by ordinary PTS technique but instead is based on a proper selection of the different subblocks. By interleaving a data frame, the peaks in the related OFDM signal can be compressed. It has been shown that the PAPR performance of modified PTS can be improved by using interleaved subblock partition scheme. Simulations results show that the modified PTS combined with interleaving technique is an effective method to reduce the PAPR of an OFDM signal.

\section{REFERENCES}

[1] R V. Nee and R. Prasad, OFDM for Wireless Multimedia Communications, London, Artech House, 2000.

[2] H. J. Taha and M. F. M. Salleh, "Multi-carrier transmission techniques for wireless communication systems: A survey," WSEAS Transactions on Communications, vol. 8, no. 5, pp. 457-472, May 2009.

[3] I. Baig and V. Jeoti, "A novel precoding based hybrid MC/SC radio access system for PAPR reduction in layered OFDMA of LTE-Advanced," IAENG International Journal of Computer Science, vol. 38, no. 4, pp. 314-319, November 2011.

[4] S. K. Deng and M. C. Lin, "Recursive Clipping and Filtering with bounded distortion for PAPR reduction," IEEE Transactions on Communication, vol. 55, no. 1, pp. 227-230, January 2007.

[5] G. Lu, P. Wu, and C. C. Logothetis, "Enhanced Interleaving partitioning PTS for peak-to-average power ratio reduction in OFDM systems," IEEE Electronics Leters, vol. 42, no. 17, pp. 983-984, August 2006.

[6] R. Baumal, R. Fischer, and J. Huber, "Reducing the peak-to-average power ratio of multicarrier modulation by selective mapping," IEEE Electronics Letters, vol. 32, no. 22, pp. 2056-2057, October 1996.

[7] L. J. Cimini and N. R. Sollenberger, "Peak-to-average power ratio reduction of an OFDM signal using Partial Transmit Sequences," IEEE Communications Letters, vol. 4, no. 3, pp. 86-88, March 2000.

[8] Z. Xiuyan and T. Guobin, "The research of improved PTS method for peak-to-average power ratio reduction," in Proc. of IEEE 3rd international conference on wireless, mobile and multimedia networks, Beijing, China, September 2010, pp. 104-107.
[9] X. C. Wu, Z. G. Mao, J. X. Wang, and B. Zho, "A novel PTS technique with combinative optimization in real part and imaginary part for PAPR reduction in OFDM systems," in Proc. of Third International Conference on Next Generation Mobile Applications, Services and Technologies, Cardiff, Wales, UK, September 2009, pp. 215-218.

[10] C. Tellambura, "Phase optimization criterion for reducing peak-to-average power ratio of an OFDM signal," IEEE Electronics Letters, vol. 34, no. 2, pp. 169-170, January 1998.

[11] A. D. S. Jayalath, C. Tellambura, and H. Wu, "Reduced complexity PTS and new phase sequences for SLM to reduce PAP of an OFDM signal," in Proc. of IEEE 51st Vehicular Technology Conference, Tokyo, Japan, 2000, pp. 1914-1917.

[12] J. H. Wen, S. H. Lee, Y. F. huang, and H. L. Hung, "A suboptimal PTS algorithm based on particle swarm optimization technique for PAPR reduction in OFDM systems," EURASIP Journal on Wireless Communications and Networking, vol. 2008, Article ID 601346, pages1-8, doi:10.1155/2008/601346.

[13] A. D. S. Jayalath and C. Tellambura, "The use of Interleaving to reduce the peak-to-average power ratio of an OFDM signal," in Proc. of Global Tele communications Conference, San Francisco, CA, December 2000, pp. 82-86.

[14] S. G. Kang and J. G. Kim, "A novel subblock partition scheme for partial transmit sequence OFDM," IEEE Transactions on broadcasting, vol. 45, no. 3, pp. 333-338, September 1999.

[15] J. Sarawong, T. Mata, P. Boonsrimuang, and H. Kobayashi, "Interleaved partitioning PTS with new phase factors for PAPR reduction in OFDM systems," in Proc. of 8th International Conference on Electrical Engineering / Electronics, computer, Telecommunications and Information Technology, Khon Kaen, Thailand, May 2011, pp. 361-364.

[16] U. Sorger, I. D. Broeck, and M. Schnell, "Interleaved FDMA - A new Spread-Spectrum Multiple-Access scheme," in Proc. of International Conference on Communications, Atlanta, Georgia, USA, June 1998, vol. 2, pp. 1013-1017.

[17] A. Shukla, R. Sapra, V. Goyal, and M. Shukla, "Performance analysis of PAPR reduction in helical Interleaved OFDM system," in Proc. of International Conference on Communication systems and Network Technologies, GLA University, Mathura, India, 11-13 May 2012, pp. 639-642.

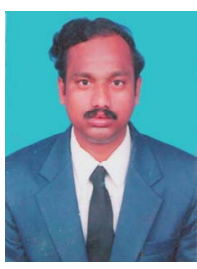

P. Mukunthan received B.E degree in Electronics and Communication Engineering from University of Madras, Chennai in 1998 and M.E degree in Applied Electronics from University of Madras, Chennai in 2000. He is working as Assistant Professor in the Department of Computer Science and Engineering, Adhiparasakthi Engineering College, Melmaruvathur, India. He is pursuing his Ph.D. in the Department of Electronics and Communication Engineering, Pondicherry Engineering College, Pondicherry, India. His research interests include digital communication, computer networks, and wireless cellular mobile network.

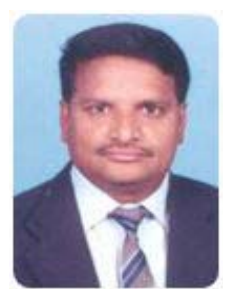

P. Dananjayan received Bachelor of Science from University of Madras in 1979, Bachelor of Technology in 1982 and Master of Engineering in 1984 from the Madras Institute of Technology, Chennai and Ph.D. degree from Anna University, Chennai in 1998. He is working as Professor in the Department of Electronics and Communication Engineering, Pondicherry Engineering College, Pondicherry, India. He has been as visiting professor to AIT, Bangkok. He has to his credit more than 90 publications in National and International Journals. He has presented more than 160 papers in National and International Conferences. He has guided $12 \mathrm{Ph} . \mathrm{D}$ candidates and is currently guiding $7 \mathrm{Ph} . \mathrm{D}$ students. His research interests include spread spectrum techniques, wireless communication, wireless adhoc and sensor networks. 